\title{
Time Course of Catch-up in Adiposity Influences Adult Anthropometry in Individuals Who Were Born Small for Gestational Age
}

\author{
NADIA EZZAHIR, CORINNE ALBERTI, SAMIA DEGHMOUN, ISABELLE ZACCARIA, \\ PAUL CZERNICHOW, CLAIRE LÉVY-MARCHAL, AND DELPHINE JAQUET \\ INSERM U457 [N.E., S.D., P.C., C.L.-M., D.J.] and Center of Clinical Epidemiology [N.E., C.A., I.Z.], \\ Robert Debré Hospital, 75019 Paris, France
}

\section{ABSTRACT}

gain in BMI during childhood. However, this effect was significant only when this gain persisted after the first year of life. Similarly, the influence of the magnitude in gain in BMI on the risk for adult BMI $>25 \mathrm{~kg} / \mathrm{m}^{2}$ was significantly influenced by the age at which the gain in BMI occurred. In summary, although the extent of catch-up in BMI affects adiposity in adulthood, this effect is mostly deleterious when occurring after 1 y of age, suggesting that a rapid catch-up process should be more suitable than a delayed one. Whether this observation holds through regarding the metabolic syndrome remains to be elucidated. (Pediatr Res 58: 243-247, 2005)

BMI, body mass index
IP, ponderal index
SGA, small for gestational age
Catch-up growth is a key physiologic process for a normal final height in individuals who were born small for gestational age (SGA) (1,2). However, this complex phenomenon is associated with substantial change in body distribution. Individuals who were born SGA display a peculiar growth pattern of adiposity: severely reduced at birth $(3,4)$, adiposity dramatically increases during the catch-up growth period in children who were born SGA, as evidenced by the noticeably increased body mass index (BMI) during infancy $(5,6)$. Furthermore, it has been shown that children who were born SGA and displayed catch-up growth during infancy showed an increased body fat mass with a more central fat distribution in comparison with children who were born with normal birth size (6). This increased growth velocity of adiposity persists until adult-

Received October 29, 2004; accepted January 3, 2005.

Correspondence: Delphine Jaquet, M.D., INSERM Unité 457, Hôpital Robert Debré, 48, boulevard Sérurier, 75019 Paris, France; e-mail: delphine.jaquet@ rdebre.inserm.fr.

This study was supported by grants from Pharmacia-Upjohn and the Institut National de la Santé et de la Recherche Médicale (INSERM, "PROGRES" 2000-2002).

DOI: 10.1203/01.PDR.0000169980.35179.89 hood, as evidenced by relative increase in BMI (expressed in SDS) from childhood to adulthood, leading to a significantly increased percentage of body fat mass at the age of $25 \mathrm{y}$ in individuals who were born SGA in comparison with control subjects $(7,8)$. Catch-up growth has been suspected to favor later development of long-term metabolic complications associated with reduced fetal growth (9-12). However, not only the extent but also the dynamic changes during childhood in adiposity in individuals who were born SGA may play a crucial role in the determination of the long-term metabolic outcome. Thus, the aims of the present study were 1) to determine the dynamic profiles of adiposity between birth and $6 \mathrm{y}$ of age in individuals who were born SGA, 2) to investigate their relationship with neonatal characteristics, and 3) to evaluate its long-term effect on adult anthropometry. To achieve these goals, we studied postnatal change in BMI from birth to $6 \mathrm{y}$ of age in terms of magnitude and time course in 136 individuals who were born SGA and selected from a maternity registry. Then, we investigated how postnatal change in BMI might influence anthropometric characteristics in adulthood. 


\section{METHODS}

Participants. Participants were selected from a previously constituted cohort in which all participants were studied at the age of $20 \mathrm{y}$ (13). Participants of this cohort were identified from a maternity registry of the metropolitan area of the city of Haguenau in France. Inclusion criteria in the cohort were singletons who were born between 32 and $42 \mathrm{wk}$ of gestation and had SGA defined as a birth weight $<10$ th percentile for gender and gestational age according to the local growth standard curves built (27,366 births). Gestational age was determined from the last menstrual period and confirmed by physical examination and ultrasonography when available. Inclusion criteria for the present study were 1) gestational age $\geq 37$ wk of gestation and 2) available serial anthropometric data at 1,2,4, and $6 \mathrm{y}$ of age and at $20 \mathrm{y}$ of age. From the 513 individuals who were born SGA and included in the cohort in 2000 and for whom anthropometric data during childhood have been recorded, 478 were born full term. Among these 478 individuals who were born SGA, 127 had available serial anthropometric data during childhood. No significant differences were observed between individuals who participated in the present study and those who did not participate in terms of gestational age, gender, birth weight, and birth length (Table 1).

Study design. Birth characteristics were derived from the registry. Postnatal individual body weight and height values at $1,2,4$, and 6 y of age were collected from each individual health leaflet. Measurements were performed during a medical visit by a physician. Body weight was measured undressed using a portable scale. Body length was measured in supine position until the age of $2 \mathrm{y}$ and standing with a wall-mounted stadiometer thereafter. Adult clinical characteristics were measured at the time of the study. Body weight was measured using an electronic scale. Body height was measured twice with a wall-mounted stadiometer, and the average value was retained in the analysis. Waist circumference was measured at the level of the umbilicus, and hip circumference was measured at the level of the greater trochanter. The study protocol was reviewed and approved by the ethical committee of the University of Paris-St. Louis, and all participants gave written informed consent.

Studied parameters. Total adiposity at birth was estimated by ponderal index (IP: $\mathrm{kg} / \mathrm{m}^{3}$ ) and BMI $\left(\mathrm{kg} / \mathrm{m}^{2}\right.$ ). Total adiposity from $1 \mathrm{y}$ to adulthood was estimated by BMI. Regional distribution of adiposity in adulthood was estimated by the waist-to-hip ratio.

Considering the respective variability of the measurements, dynamic change in BMI was considered to be significant when the slope of change in BMI between two time points from birth to $6 \mathrm{y}$ of age allowed a magnitude of gain of at least $0.3 \mathrm{SD}$. Accordingly, the age at the end of significant gain in BMI was the last time point for which the difference in BMI between this point and the previous one was $>0.3 \mathrm{SD}$. The magnitude of gain in BMI was calculated using the difference in BMI (SDS) over the period for which this change was significant. Catch-up therefore was observed when BMI was $>-1.64 \mathrm{SD}$ at any point in time (corresponding to the crude value of BMI $<10$ th percentile) and when dynamic change in BMI between two time points was significant.

Adiposity rebound is a physiologic process characterized by the spontaneous increase of adiposity, after the physiologic decrease usually observed between the age of 1 and 4-6 y (14). A precocious adiposity rebound is known to be associated with an increased risk for obesity in adulthood (14). Therefore, the time point after the age of $1 \mathrm{y}$ with the lowest individual crude value of BMI gave the age at adiposity rebound.

Statistical analysis. Statistical analyses were conducted with the use of SAS Software version 8.02 (SAS Institute, Cary, NC). IP at birth and BMI at each age point were transformed into SDS, i.e. the number of SDs by which one measure differs from the mean for a control population. A control group was used as a reference population for anthropometric data and was composed by aged-matched individuals who were born appropriate for gestational age (AGA) and selected from the same population-based registry, including 574

Table 1. Birth characteristics of participants and non-participants in the present study

\begin{tabular}{lccc}
\hline & $\begin{array}{c}\text { Participants } \\
(n=136)\end{array}$ & $\begin{array}{c}\text { Non participants } \\
(n=351)\end{array}$ & $p$ \\
\hline Gender (M/F) & $62 / 65$ & $156 / 195$ & 0.41 \\
Gestational age (wk) & $39(37 ; 42)$ & $39(37 ; 42)$ & 0.41 \\
Birth weight $(\mathrm{g})$ & $2750(1780 ; 3080)$ & $2750(1660 ; 3080)$ & 0.40 \\
Birth length $(\mathrm{cm})$ & $48(42 ; 52)$ & $48(40 ; 52)$ & 0.73 \\
\hline
\end{tabular}

Results are given as median (range). $p$ values for anthropometric data are given after adjustment for gender and gestational age. singletons who were born full term, with birth size between the 25 th and the 75 th percentiles and with available anthropometric data at birth and 1,2, 4, and $6 \mathrm{y}$ of age.

SDS at birth and in childhood were derived from the population that was born appropriate for gestational age; age-specific reference intervals for childhood anthropometric parameters were estimated by the simplified parametric method of Royston and Wright (15), in which fractional polynomials in age are fitted simultaneously to median, scale, and skewness of an exponential normal distribution by maximum likelihood.

As the childhood anthropometric data were collected in a retrospective manner in the health leaflet, we tolerated only the values at $4 \mathrm{y}$ of age to be missing, considering the relatively stable evolution of BMI between 2 and $6 \mathrm{y}$. Missing data were defined "missing at random" and imputed using multiple imputation (PROC MI, SAS). Missing weight and height data at 4 y were imputed at first with 10 iterations (16). BMI at $4 \mathrm{y}$ was calculated thereafter using the imputed data. Quantitative variables were expressed as median (range) because of skewed distributions. Qualitative variables were expressed as frequency (percentages). Comparisons used $\chi^{2}$ or Fischer exact tests where appropriate for qualitative variables and nonparametric ANOVA for quantitative variables. Comparisons of quantitative variables between the groups were performed using the Kruskall-Wallis nonparametric test. The association between two quantitative variables was assessed by the correlation coefficient of Spearman. The relationships between childhood growth parameters and adult anthropometric data were evaluated using the generalized linear model with adjustment on age and gender when necessary. For each model, normality of the residuals distribution and absence of heteroscedasticity were checked. Results of the models were expressed by the slope parameter and its $p$ value. A $p \leq 0.05$ was considered to indicate statistical significance.

\section{RESULTS}

Time course and magnitude of change in BMI and adiposity rebound during childhood. Figure 1 illustrates the evolution of BMI from birth to $6 \mathrm{y}$ of age. At birth, median BMI was -2.2 SD (-4.9-0.4). Thinness at birth in the SGA group was followed by a significant increase in BMI for 115 participants (91\%), terminated after the first $2 \mathrm{y}$ of life in most participants. Twelve (9\%) participants did not display any significant change in BMI or remained with a BMI below $-1.64 \mathrm{SD}$ over the study period and were considered as having no catch-up in BMI. These participants did not significantly differ from those with catch-up in terms of gestational age [39 wk (37-42) versus 39 wk (37-42); $p=0.51]$, IP [ $-0.9 \mathrm{SD}$ $(-3.0-0.7)$ versus $-1.0 \mathrm{SD}(-4.0-2.3) ; p=0.55]$, and BMI (Fig. 2). However, they were significantly leaner at $6 \mathrm{y}$ of age (Fig. 2).

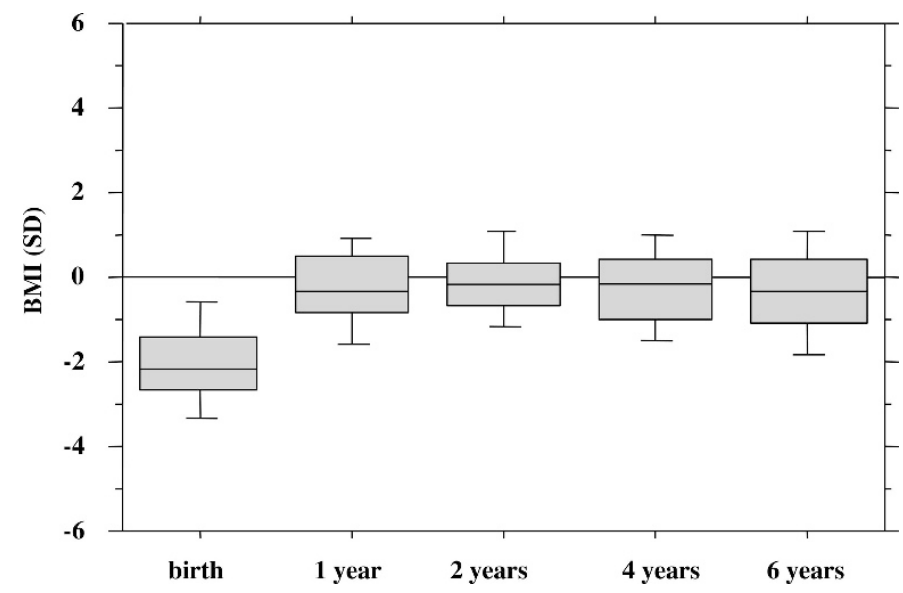

Figure 1. Changes in BMI from birth to $6 \mathrm{y}$ of age in participants who were born SGA $(n=127)$. BMI is expressed in SDS of the reference population. Data are represented as box plots with median, 25th and 75th, and 10th and 90th percentiles. 


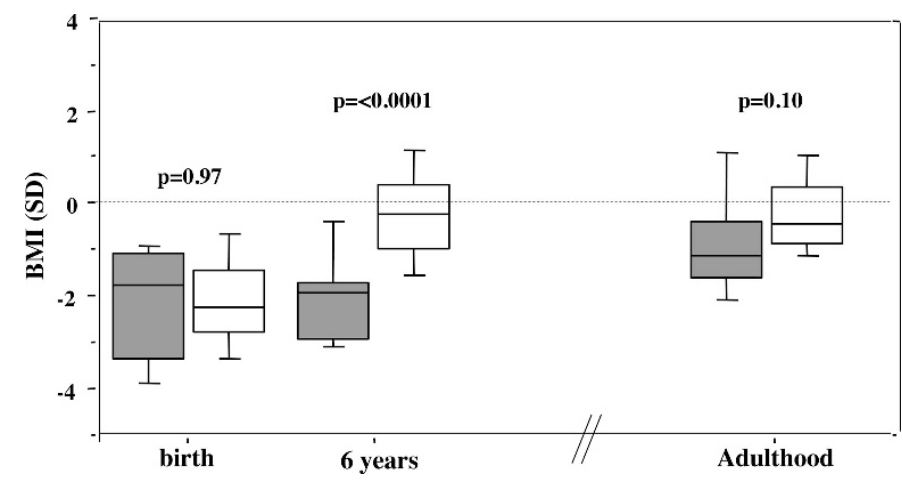

Figure 2. Changes in BMI from birth to adulthood in participants who were born SGA according to catch-up in BMI status during childhood. BMI is expressed in SDS of the reference population. Data are represented as box plots with median, 25th and 75th, and 10th and 90th percentiles. $\square$, Participants who showed no catch-up in childhood; $\square$, participants with catch-up in BMI. Statistical analyses were performed with the Mann-Whitney nonparametric tests.

Among the 115 participants who were born SGA with significant increase in BMI, the median magnitude of this change was $2.5 \mathrm{SD}(0.4-7.41)$. This magnitude was inversely and significantly correlated with IP $(\rho=-0.55, p<0.0001)$ and BMI at birth $(\rho=0.63, p<0.0001)$. Dynamic change in BMI was no longer significant after the first year of life in 61 (53\%) participants, after 2 y of age in 43 (34\%) participants, after 4 y of age in $11(10 \%)$ participants, but was still observed at $6 \mathrm{y}$ of age in $4(3 \%)$ participants. Ten of 115 participants displayed negative change in BMI or no change at all within the first year of life. Among these participants, five experienced catch-up during the second year, two displayed significant change in BMI between 2 and 4 y of age, and three displayed significant change in BMI after the age of 4 only. As indicated in Table 2, the time course of dynamic change in BMI was not influenced by thinness at birth and did not significantly influence the magnitude of gain in BMI as well as the 6-y BMI.

Among the 127 participants who were born SGA, adiposity rebound was observed at $2 \mathrm{y}$ of age in $10(8 \%)$ participants, at $4 \mathrm{y}$ of age in $50(39 \%)$ participants, and at $6 \mathrm{y}$ of age in 67 $(53 \%)$ participants (Table 3 ). The age at adiposity rebound was not influenced by the existence of catch-up between 0 and $6 \mathrm{y}$ of age $(p=0.50)$. However, early adiposity rebound ( $2 \mathrm{y}$ of age) was associated with thinness at birth, greater magnitude of change in BMI, and larger BMI at $6 \mathrm{y}$ of age (Table 3). Moreover, early adiposity rebound was observed in four of 15
(27\%) participants for whom significant changes in BMI were still observed after the age of $2 \mathrm{y}$, in comparison with only five $(8 \%)$ of 61 participants with change in BMI during the first year only ( $p=0.006$ ). Gender had no significant influence on postnatal change in BMI in terms of either magnitude or time course.

Consequences on adult anthropometric parameters. The mean adult age of the study population was $20.1 \pm 2.8 \mathrm{y}$. Thinness at birth expressed by IP or BMI did not affect adult BMI $(\rho=-0.03, p=0.69 ; \rho=0.04, p=0.63$, respectively) or adult waist-to-hip ratio adjusted for age and gender $(\beta=$ $0.007, p=0.29 ; \beta=0.0002, p=0.83$, respectively). Whereas the 6-y BMI was significantly reduced in participants who did not catch up in BMI during early childhood, the difference in adult BMI between participants with or without catch-up was reduced and did not reach statistical significance (Fig. 2). Adult BMI was significantly correlated with the magnitude of gain in BMI in childhood ( $\rho=0.18, p=0.05)$ but was not related to the time course of change in BMI (Table 2). However, the effect of the magnitude of gain in BMI on adult BMI tended to differ according to this time course $(p=0.08)$; more specific, the magnitude of gain in BMI significantly influenced adult BMI only when changes in BMI occurred or were still significant after the first year of life (Fig. 3).

As expected, an early adiposity rebound was significantly associated with higher BMI in adulthood (Table 3). The effect of the magnitude of gain in BMI on adult BMI was not significantly influenced by the age at adiposity rebound ( $p=$ 0.60).

Among the 127 participants, 19 were overweighed (BMI $\left.>25 \mathrm{~kg} / \mathrm{m}^{2}\right)$ and four were obese $\left(>30 \mathrm{~kg} / \mathrm{m}^{2}\right)$ as young adults. The risk for having a BMI $>25 \mathrm{~kg} / \mathrm{m}^{2}$ was not influenced by the existence of catch-up in BMI during childhood ( $p=0.34$ ) and the time course of change in BMI $(p=0.40)$ but was significantly associated with early adiposity rebound (odds ratio $0.7 ; 95 \%$ confidence interval $0.5-1.0 ; p=0.04)$. The magnitude of gain in BMI from birth to $6 \mathrm{y}$ of age was positively associated with the risk for being overweight in adulthood (odds ratio 1.9; 95\% confidence interval 1.2-3.0; $p$ $=0.007)$; however, here again this effect significantly differed according to the time course of change in BMI $(p=0.05)$ and became significant only when occurring after the age of $1 \mathrm{y}$.

Dynamic change in BMI both in terms of time course and in terms of magnitude did not influence adult waist-to-hip ratio

Table 2. Adiposity at birth, during childhood and adulthood according to age at the end of change in BMI

\begin{tabular}{|c|c|c|c|c|}
\hline & $1 \mathrm{yr}(n=61)$ & $2 \operatorname{yrs}(n=39)$ & $4-6 \operatorname{yrs}(n=15)$ & $p$ \\
\hline Gestational age (wk) & $39(37 ; 42)$ & $40(37 ; 42)$ & $39(37 ; 41)$ & 0.02 \\
\hline Ponderal Index (SD) & $-0.58(-2.43 ; 1.54)$ & $-0.78(-1.78 ; 0.77)$ & $-0.24(-1.81 ; 1.26)$ & 0.72 \\
\hline BMI at birth (SD) & $-2.2(-4.9 ; 0.4)$ & $-2.2(-4.0 ; 0.0)$ & $-1.6(-3.3 ; 0.4)$ & 0.39 \\
\hline$\Delta \mathrm{BMI}(\mathrm{SD})$ & $2.4(0.3 ; 4.5)$ & $2.6(0.5 ; 5.4)$ & $3.0(0.6 ; 7.1)$ & 0.07 \\
\hline 6 yrs BMI (SD) & $-0.3(-2.2 ; 2.5)$ & $-0.2(-2.1 ; 4.9)$ & $0.1(-1.6 ; 3.1)$ & 0.08 \\
\hline Adult BMI (SD) & $-0.4(-1.6 ; 3.0)$ & $-0.4(-1.6 ; 4.2)$ & $-0.3(-1.6 ; 1.5)$ & 0.53 \\
\hline Adult W/H & $0.78(0.68 ; 0.98)$ & $0.78(0.65 ; 1.06)$ & $0.83(0.67 ; 0.91)$ & $0.99 *$ \\
\hline
\end{tabular}

Ponderal index and BMI are expressed in standard deviation score of the reference population (SD).

$\Delta$ BMI = magnitude in BMI during catch-up growth (BMI (SD) at the end of catch-up-BMI (SD) at birth). W/H: waist-to-hip ratio. Results are expressed as median (range).

* $p$ value was obtained after adjustment for gender and age. 
Table 3. Adiposity at birth, childhood and adulthood according to age at adiposity rebound

\begin{tabular}{|c|c|c|c|c|}
\hline & $2 \mathrm{yr}(n=9)$ & $4 \mathrm{yrs}(n=49)$ & 6 yrs $(n=69)$ & $p$ \\
\hline Gestational age (wk) & $39(37 ; 41)$ & $39(37 ; 42)$ & $40(37 ; 42)$ & 0.8 \\
\hline Ponderal Index (SD) & $-1.7(-4.0 ;-0.1)$ & $-0.9(-3.4 ; 2.3)$ & $-1.1(-2.9 ; 2.3)$ & 0.08 \\
\hline BMI at birth (SD) & $-2.6(-4.9 ; 1.4)$ & $-1.9(-4.9 ; 0.4)$ & $-2.2(-3.7 ; 0.2)$ & 0.02 \\
\hline 6 yrs BMI (SD) & $2.0(0.1 ; 3.2)$ & $0(-1.6 ; 4.9)$ & $-0.6(-2.2 ; 2.0)$ & $<0.0001$ \\
\hline Adult BMI (SD) & $0.3(-0.4 ; 1.5)$ & $-0.5(-1.6 ; 4.2)$ & $-0.5(-1.6 ; 2.8)$ & 0.05 \\
\hline Adult W/H & $0.77(0.68 ; 0.86)$ & $0.79(0.69 ; 0.98)$ & $0.79(0.65 ; 1.06)$ & $0.69^{*}$ \\
\hline
\end{tabular}

Ponderal index and BMI are expressed in standard deviation score of the reference population (SD).

$\Delta$ BMI = magnitude in BMI during catch-up growth (BMI (SD) at the end of catch-up-BMI (SD) at birth). W/H: waist-to-hip ratio. Results are expressed as median (range).

* $p$ value was obtained after adjustment for gender and age.
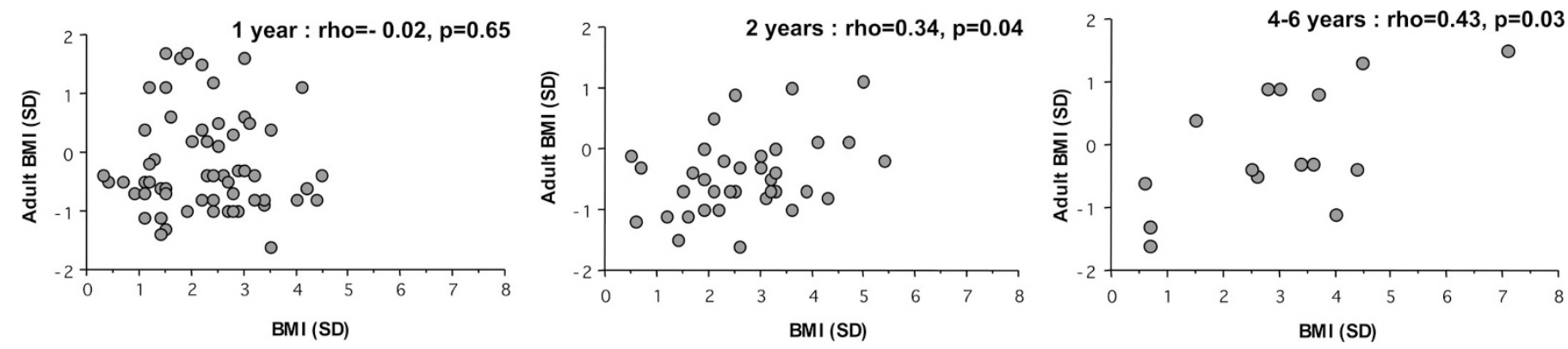

Figure 3. Relationship between the magnitude of catch-up in BMI during childhood and adult BMI according to age at the end of catch-up. BMI is expressed in SDS of the reference population. Magnitude of catch-up in BMI was expressed as the difference $(\Delta)$ between the BMI (SD) at birth and the BMI (SD) at the end of catch-up. Statistical analyses were performed using the Spearman correlation test.

when age and gender were taken into account (Table 2). Similarly, age at adiposity rebound did not influence the adult waist-to-hip ratio (Table 3).

\section{DISCUSSION}

As expected, individuals who were born SGA display significant change in BMI during infancy and early childhood to compensate for thinness at birth $(5,6)$. It is noteworthy that the spontaneous change in BMI leads to a BMI within the normal range during childhood and adulthood for the majority of the study population, reflecting, therefore, a catch-up process. However, this catch-up is far more pronounced during the first year of life, which is in agreement with previous data demonstrating that weight catch-up precedes length catch-up in children who were born SGA and that the majority of weight deficiency is corrected during the first months of life, whereas length catch-up is more gradual and prolonged until the age of $2-3$ y $(2,17)$. However, in our study population, $9 \%$ of children did not show any catch-up in BMI from birth to $6 y$ of age. This lack of catch-up was unexpected because these children were born with comparable thinness as those with catch-up. Consequently, children with no catch-up were severely thin at $6 \mathrm{y}$ of age. No significant association was found between lacks of catch-up in BMI and gestational age in the present study. Therefore, the reason for this lack of catch-up might be linked to the process that leads to thinness at birth during prenatal life or to events that occur during early childhood, such as feeding behavior.

According to the time course of changes in BMI observed in this study population, changes in BMI were no more significant after $1 \mathrm{y}$ of age in more than one of two participants who were born SGA. However, participants for whom gain in BMI ended later did not necessarily display a prolonged catch-up. Indeed, $19 \%$ of them showed an initial failure to thrive during the first 1 or $2 \mathrm{y}$ of life. It is interesting that the later the end of gain in BMI occurred, the greater was the proportion of participants in whom significant change in BMI occurred after $1 \mathrm{y}$ of age. Consequently, an older age at the end of significant change in BMI does not necessary reflect results from a persistent weight gain after the catch-up growth period but rather from a delayed catch-up process. It is interesting that the mean adult BMI in the group of participants who did not catch up during childhood did not significantly differ from those who showed catchup, although they were extremely thin at $6 \mathrm{y}$ of age, suggesting once again a delayed catch-up growth process, which likely occurs during puberty. This hypothesis was strengthened by several observations of significant weight and height gain between the ages of 8 and $14 \mathrm{y}$ in individuals who were born with extremely low birth weight $(18,19)$. What influences the time course of postnatal gain in BMI still remains unclear, because it is not influenced by body proportion at birth. Moreover, no significant association was found between this time course and the magnitude of gain in BMI, suggesting that factors that control for the magnitude of change in BMI are independent of the timing of this process.

One of our aims was to investigate the consequences of postnatal changes in BMI on body proportion in adulthood. We demonstrate in the present study that this process influences adult anthropometry. The magnitude of catch-up in BMI occurring in early childhood was significantly correlated with 
adult BMI. This observation is in agreement with previous data demonstrating that catch-up in weight during childhood was associated with obesity later in life $(6,9)$. Our results also suggest that the magnitude of catch-up increases the risk for obesity in individuals who were born SGA. This therefore would explain the association between restricted fetal growth and adult obesity occasionally reported (20).

The more striking observation is that the detrimental effect of the extent of gain in BMI during childhood on adult adiposity is visible only when postnatal change in BMI is prolonged or delayed. This effect is even blunted in individuals who displayed no more significant change in BMI after the age of $1 \mathrm{y}$. This observation is supported by the study of Bhargava et al.(21), who demonstrated in an Indian population that low BMI during the first $2 \mathrm{y}$ of life was followed by an accelerated increase in BMI until adulthood that leads to increased adiposity in early adulthood, whereas no significant association was clearly evidenced with thinness at birth alone $(21,22)$. Taken together, these observations strongly suggest that catch-up in $\mathrm{BMI}$ is not necessarily deleterious, provided that it occurs early during infancy. An attractive hypothesis might explain this clinical observation. Normally, BMI dramatically increases during the first year of life, whereas it decreases thereafter until the adiposity rebound, which generally occurs between the ages of 4 and 6 y (14). It has been shown that early adiposity rebound ( 2 y of age), which reflects an increased BMI during a period normally characterized by a decreased or stable BMI, has adverse anthropometric and metabolic effects in adulthood $(21,22)$. Similarly, we observed that gain in BMI after 2 y of age seems to have adverse effects in adulthood; it therefore can be speculated that catch-up in BMI might not be deleterious when restricted to a period of spontaneous increase in adiposity.

No significant association between thinness at birth and adult adiposity at the age of $20 \mathrm{y}$ could be observed in this study population, yet it must be pointed out that thinness at birth was associated with earlier adiposity rebound, which increases adult BMI and the risk for obesity on the one hand and was correlated with the magnitude of gain in BMI that also favors adult adiposity and obesity on the other hand. This observation holds true whether thinness at birth is estimated by BMI, which allows a similar estimate throughout the study period, or by IP, which is more appropriate to newborns. This suggests that the prenatal process that leads to thinness at birth might have a strong influence on the postnatal growth pattern and emphasizes that this process must be taken into account to understand fully the long-term effect of the catch-up growth process.

\section{CONCLUSION}

In summary, we have shown in our study population that the extent of postnatal gain in BMI in early childhood affects adiposity in adults who were born SGA. However, the magni- tude of this effect is strongly influenced by the age at which it takes place. Moreover, our results strongly suggest that clinical practitioners should not fear a rapid catch-up growth process but rather a delayed one. Whether this observation would also apply to the metabolic syndrome and, more specific, to insulinresistance associated with restricted fetal growth remains to be elucidated.

Acknowledgments. We acknowledge the contribution of C. Traband, M. Grolet, and M. Cominotti (registered nurses) at the hospital of the city of Haguenau and I. Evrard at the Center of Clinical Epidemiology of the Robert Debré Hospital.

\section{REFERENCES}

1. Karlberg J, Albertsson-Wikland K 1995 Growth in full-term small-for-gestational-age infants: from birth to final height. Pediatr Res 38:733-739

2. Albertsson Wikland K, Wennergren G, Wennergren M, Vibergsson G, Rosberg S 1993 Longitudinal follow-up of growth in children born small for gestational age. Acta Paediatr 82:438-443

3. Petersen S, Gotfredsen A, Knudsen FU 1988 Lean body mass in small for gestational age and appropriate for gestational age infants. J Pediatr 113:886-889

4. Enzi G, Zanardo V, Caretta F, Inelmen EM, Rubaltelli F 1981 Intrauterine growth and adipose tissue development. Am J Clin Nutr 34:1785-1790

5. Jaquet D, Léger J, Tabone MD, Czernichow P, Lévy-Marchal C 1999 High serum leptin concentrations during catch-up growth of children born with intra-uterine growth retardation. J Clin Endocrinol Metab 84:1949-1953

6. Ong KK, Ahmed ML, Emmett PM, Preece MA, Dunger DB 2000 Association between postnatal catch-up growth and obesity in childhood: prospective cohort study. BMJ 320:967-971

7. Leger J, Limoni C, Collin D, Czernichow P 1998 Prediction factors in the determination of final height in subjects born small for gestational age. Pediatr Res 43:808812

8. Jaquet D, Gaboriau A, Czernichow P, Levy-Marchal C 2001 Relatively low serum leptin levels in adults born with intra-uterine growth retardation. Int J Obes Relat Metab Disord 25:491-495

9. Ong KK, Dunger DB 2002 Perinatal growth failure: the road to obesity, insulinresistance and cardio-vascular disease in adults. Best Pract Res Clin Endocrinol Metab 16:191-207

10. Valdez R, Athens MA, Thompson GH, Bradshaw BS, Stern MP 1994 Birthweight and adult health outcomes in a biethnic population in the USA. Diabetologia 37:624-631

11. Eriksson JG, Forsen T, Tuomilehto J, Jaddoe VW, Osmond C, Barker DJ 2002 Effects of size at birth and childhood growth on the insulin resistance syndrome in elderly individuals. Diabetologia 45:342-348

12. Lithell HO, McKeigue PM, Berglund Mohsen R, Little UB, Leon DA 1996 Relation of size at birth to non-insulin dependent diabetes and insulin concentrations in men aged 50-60 years. BMJ 312:406-410

13. Jaquet D, Collin D, Lévy-Marchal C, Czernichow P 2004 Adult height distribution in subjects born small for gestational age. Horm Res 62:92-96

14. Rolland-Cachera MF, Deheeger M, Bellisle F, Sempé M, Guilloud-Bataille M, Patois E 1984 Adiposity rebound in children: a simple indicator for predicting obesity. Am J Clin Nutr 39:129-135

15. Wright EM, Royston P 1997 Simplified estimation of age-specific reference intervals for skewed data. Stat Med 16:2785-2803

16. Barzi F, Woodword M 2004 Imputations of missing values in practice: results from imputations of serum cholesterol in 28 cohort studies. Am J Epidemiol 160:34-45

17. Leger J, Limoni C, Czernichow P 1997 Prediction of the outcome of growth at 2 years of age in neonates with intra uterine growth retardation. Early Hum Dev 48:211-223

18. Ford GW, Doyle LW, Davis NM, Callanan C 2000 Very low birth weight and growth into adolescence. Arch Pediatr Adolesc Med 154:778-784

19. Doyle LW, Faber B, Callanan C, Ford GW, Davis NM 2004 Extremely low birth weight and body size in early adulthood. Arch Dis Child 89:347-350

20. Ravelli GP, Stein ZA, Susser MW 1976 Obesity in young men after famine exposure in utero and early infancy. N Engl J Med 295:349-353

21. Bhargava SK, Sachdev HS, Fall CH, Osmond C, Lakshmy R, Barker DJ, Biswas SK Ramji S, Prabhakaran D, Reddy KS 2004 Relation of serial changes in childhood body-mass index to impaired glucose tolerance in young adulthood. N Engl J Med 350:865-875

22. Eriksson JG, Forsen T, Tuomilehto J, Osmond C, Barker DJ 2003 Early adiposity rebound in childhood and risk for type-2 diabetes in adult life. Diabetologia 46:190194 